\title{
The more, the better?: Number of decisions as a determinant of memorability
}

\author{
BRIAN H. ROSS \\ Stanford University, Stanford, California 94305
}

\begin{abstract}
Three experiments tested alternative explanations to the Johnson-Laird, Gibbs, and deMowbray (1978) proposal that the number of decisions made about a word is a major determinant of its memorability. The alternatives considered were increased processing time, retrieval aids, and the number of positive decisions. In Experiment 1, subjects made a speeded classification about each word for a category defined by three properties. Though items with more target properties were processed longer and better remembered, within a property level processing time was not associated with better memory. In Experiment 2, subjects received three properties, but different groups responded "Yes" to words with all three properties, words with any of the three properties, or whether words had each of the properties. As predicted by the number-of-decisions hypothesis, but not by the other hypotheses, with an increasing number of target properties, recall generally increased in the ALL group, decreased in the ANY group, and remained constant in the EACH group. In Experiment 3, this recall pattern was replicated with a different set of two target properties. The number-of-decisions notion is contrasted to the elaboration principle, and a simple model is proposed. It is concluded that the number of decisions is a useful technique for investigating within-level encoding differences.
\end{abstract}

How well an item is remembered is strongly influenced by the perceptual and cognitive operations performed upon it. The levels-of-processing approach (Craik \& Lockhart, 1972) focuses on processes affecting memory and, particularly, on the importance of these encoding operations. The memory trace is viewed as a byproduct of perceptual analysis, with the level of the analysis being a major determinant of later memory for that item. Since the original formulation, the framework has been revised several times to accommodate new data (Craik \& Tulving, 1975; Fisher \& Craik, 1977; Moscovitch \& Craik, 1976). In these revisions, other principles of memory were proposed to augment the depth hypothesis, viz., elaboration, congruity, and distinctiveness.

Recently, several researchers have questioned the levels-of-processing approach (e.g., Baddeley, 1978; Nelson, 1977; Postman, Thompkins, \& Gray, 1978). Two criticisms are pertinent here. A major problem, mentioned in all these articles, is the circularity of the notion of depth of processing, the failure to provide any independent measure of depth. A second criticism is that little work has concentrated on variations in processing within a level, despite the obvious importance of

I thank Lawrence Barsalou, Francis Bellezza, Gordon Bower, Susan Jennings, P.N. Johnson-Laird, and Edward Smith for advice and encouragement on this project and manuscript. I also thank Ellen Mandinach and Ted Rafalovitch for their assistance and Beth Tanke and her students for their help. This research was partially supported by Grant MH-13950 from the National Institutes of Mental Health to Gordon $\mathrm{H}$. Bower and a Stanford University graduate fellowship. Address correspondence to Brian H. Ross, Department of Psychology, Stanford University, Stanford, California 94305. differences within the semantic level. The notion that elaboration, which is the richness of the trace, may be an important factor as well has been proposed, partially to deal with differences within a level. Within a given level, the more elaborated the code, the more likely it is to be remembered. There are, however, serious theoretical and assessment problems with the idea of elaboration (see Postman et al., 1978). Even when specific elaborative mechanisms have been proposed (Anderson, 1976, chap. 10), an independent measure is extremely difficult.

Johnson-Laird and his associates (Johnson-Laird \& Bethell-Fox, 1978; Johnson-Laird, Gibbs, \& deMowbray, 1978) have proposed the concept of amount of processing as an unambiguous measure for studying memory differences within a level. "Amount of processing is defined in terms of the number of decisions about an item that yield pertinent information to the task at hand" (Johnson-Laird et al., 1978, p. 374). If the number of pertinent decisions (or the ranking of conditions on this variable) can be determined, within-level differences may be investigated. In this article, this notion will be called number of decisions instead of amount of processing. (Number of decisions is only one way of operationalizing amount of processing; the latter term is so vague that a clearer, more restrictive notion may be preferable at this time.) Before discussing the Johnson-Laird et al. (1978) results, a brief review of some earlier work employing similar ideas will be given.

Ratings, Specificity, and Amount of Processing

Some research with a levels-of-processing orientation 
has investigated differences within a level by varying the number of ratings required for an item. Hyde (1973) included three semantic orienting tasks. Two groups of subjects rated items on one semantic scale, either pleasantunpleasant or active-passive, while a third group rated items along both scales. The recall performance of the three groups did not differ. Klein and Saltz (1976) examined recall for words rated on one or two scales but also manipulated the judged correlation of the two scales. They found higher recall with two dimensions than with one and, within the two-dimension data, higher recall with greater independence of dimensions.

A second group of studies has involved a more subtle manipulation of processing, namely, specificity. Frase and Kammann (1974) had subjects search lists for instances of a general category (e.g., foods) or a more specific category (e.g., vegetables). In all three of their experiments, free recall was higher following the search for specific categories. Bock (1976) included a similar manipulation and obtained the same results. Craik and Tulving (1975) conducted some related experiments in proposing the notion of elaboration. For example, in their Experiment 7, for "Yes" responses, recall increased with the complexity of the orienting sentence frame used. The logic of these studies is that to decide whether an item meets specific constraints involves more checking of features or more processing than does a decision about more general constraints.

These studies, though their theoretical motivations were slightly different, seem to support the notion that a greater number of decisions within a level results in better memory. The greater the number of decisions (ratings, feature checks) made about an item, the more likely it is to be remembered. Two highly correlated decisions may not improve memory, as in Hyde (1973) and Klein and Saltz (1976), because similar information is used for both ratings so that the additional processing of the second rating is small.

Though these studies are consistent with the notion of number of decisions, they do not provide strong support for it. One problem in interpreting the aforementioned results in terms of the number of decisions at encoding is that the number of ratings and specificity results may be retrieval effects (Johnson-Laird et al., 1978). Two rating scales may result in more possible categories to use as retrieval cues, which it is known will help recall (Mandler, 1967). A second difficulty with this work is that determining membership in specific classes does not necessarily require more decisions or processing than for more general classes (see Rips, Shoben, \& Smith, 1973). Another problem is that the use of evaluative ratings, since they do not usually involve simple fact retrieval, may involve strategies that could generate the results. For instance, Postman and Kruesi (1977) suggested that evaluative ratings involve displaced rehearsal, as subjects compare items to earlier items and their ratings. If ratings on items along similar dimensions could be made by retrieving earlier items rated along either dimension, this proposal would predict the Klein and Saltz (1976) findings. Since earlier items rated along similar dimensions could be used in both dimension's comparisons, the average number of displaced rehearsals would be fewer than for items rated along dissimilar dimensions. A final difficulty with using the number of ratings and specificity results as support for the number-of-decisions hypothesis is that these studies allowed strategies to change with experimental conditions. Specificity manipulations, for example, were varied between subjects or blocked within subjects. For a convincing demonstration that the number of decisions per se is a major determinant of an item's memorability, it is imperative to show that substantial memory performance differences may be obtained between conditions that vary only on the number of decisions made about each item.

\section{Number of Decisions}

Johnson-Laird et al. (1978) employed the notion of number of decisions to investigate whether semantic processing of a word necessarily activates all the elements of its meaning. Subjects searched for instances of target categories, defined by possessing a prespecified set of properties. Items contained all, some, or none of these properties. Assuming a self-terminating search (i.e., that subjects could reject a word as soon as one property was found to be lacking), the number of decisions, and hence the memorability, of an item should increase with the number of target properties it possesses. This result was predicted and obtained, with the proportion recalled in their Experiment 2 of .11 , .22 , and .50 for words with 0,1 , and 2 target properties, respectively.

This method obviates the criticisms leveled against the studies discussed earlier. It allows an unambiguous manipulation within the semantic level, and, since the decisions made for items with few properties are included in the decisions made for items with more properties, comparisons are straightforward. Also, objective semantic properties may be used, avoiding the possible displaced rehearsal problem of evaluative ratings. Finally, this procedure does not allow strategies to vary with the number of decisions made about an item.

The amount of processing, as operationalized in the Johnson-Laird et al. (1978) procedure, may be an important tool for investigating memorability within a level, but their experiment does not allow an unequivocal conclusion that the number of decisions is the determining factor. At least three other variables are confounded with number of decisions in their procedure.

One confounded variable is the amount of processing time taken. Johnson-Laird et al. (1978) noted this possibility, but claimed there were two pieces of evidence against it. One contrary finding was that they found the subjects' times to search the word lists did not correlate with the number of items recalled. However, the measures were total time and total recall, so that the 
low correlation shows only that fast subjects recall about the same number of items as do slow subjects. The question, then, is not whether processing time will be correlated with memorability, but whether, within a given number of decisions, greater decision time leads to greater memorability.

This issue is similar to the one addressed by Craik and Tulving (1975) in a reanalysis of their Experiment 2 (pp. 279-280). They reasoned that if processing time is a crucial determinant of memory performance, then, within a task, longer processing times should be associated with better performance. Their claim, based on their Figure 5 (p. 280), is that longer times were not associated with better memory. Two difficulties prevent applying these results to the present task as JoinnsonLaird et al. (1978) proposed. One difficulty is that, though the rhyme (e.g., "Does the word rhyme with train?") and case (e.g., "Is the word in capital letters?") conditions showed no increase in recognition memory with longer times, the sentence (semantic, e.g., "Does the word fit in 'He met a ___ on the street.'?") conditions did increase slightly for both "Yes" and "No" decisions. No statistical tests were performed, so it is unclear whether these are reliable increases in the semantic conditions. Even if these increases are not reliable (or even replicable), a purely semantic task in which fast and slow conditions differ only on the number of decisions, and not on the type of decision, may be different enough from the case-rhyme-sentence tasks to make direct extensions inadvisable.

A second confounding with the number-of-decisions hypothesis in the Johnson-Laird et al. (1978) study is a more subtle, retrieval hypothesis. Since all words for a subject were categorized with respect to the same three properties, Johnson-Laird et al. (1978) claim that variations in word recall cannot be due to the number of categories available as retrieval cues. This claim does not address other retrieval influences that would be consistent with the obtained results. Imagine that subjects are searching a list for words satisfying the three properties of being consumable natural solids (e.g., apple). When asked to recall, it is possible that subjects would use as retrieval cues the three properties they are searching for in the list. If so, almost any search strategy (especially combined over subjects) would predict that the more target properties an item contained, the more likely it would be to be recalled. For example, a subject might initially use each property separately to try to recall and, then, as the single cue's effectiveness wanes, try conjunctions of two or three properties as retrieval cues. Evidence against this explanation would be to find a situation in which items with fewer target properties require a greater number of decisions and are better recalled than are items with more target properties, or to show similar results in recognition memory where retrieval strategies may be less influential.

Another possible explanation of the Johnson-Laird et al. (1978) results is the number of positive decisions, but this was also confounded with the number of decisions. This explanation claims that decisions help only when a property of the word matches one of the target properties. Assuming a self-terminating model, JohnsonLaird et al. confounded the expected number of decisions for each word with the expected number of positive decisions. For items with $0,1,2$, and 3 target properties, the expected numbers of decisions are $1.0,1.33,2.0$, and 3.0 , respectively, while the corresponding expected numbers of positive decisions are $.0, .33,1.0$, and 3.0. Thus, if the number of decisions improves memorability only when a property of the word matches one of the target properties, the same pattern of results would be predicted. This explanation is similar to the "congruity hypothesis" (Schulman, 1974), which proposes that positive decisions generally lead to better memory. Craik and Tulving (1975, Experiment 6) suggested that the congruity effect is not due to positive decisions per se but, rather, to the likelihood of greater elaboration in those cases. They showed that when positive and negative decisions allowed what seemed to be equal amounts of elaboration (e.g., "Is this object taller than a man?"), there was no congruity effect. The positive and negative decisions in the Johnson-Laird et al. study may have allowed equal elaboration, but possibly not. More convincing evidence against the congruity hypothesis would be to show better memory performance for words requiring negative decisions.

Three alternatives to the number-of-decisions explanation have been mentioned: processing time, retrieval aids, and the number of positive decisions. The hypothesis that number of decisions determines memory performance is promising, but before it can be adopted, these alternatives must be ruled out. The experiments reported below do this. In Experiment 1, the processing time explanation is tested. In Experiments 2 and 3, the retrieval aids, number-of-positive-decisions, and the number-of-decisions hypotheses are unconfounded to determine which one best predicts memory performance.

\section{EXPERIMENT 1}

Experiment 1 examined the possibility that processing time, rather than number of decisions, was the main determinant of memory performance. The procedure was similar to that of Johnson-Laird et al. (1978), but reaction times were taken for each item rather than for the whole list. In addition, a recognition memory test was included following the unexpected free recall test.

\footnotetext{
Method

Design. Subjects were given three properties which they were told defined a category. Using these properties, they were to make speeded classification judgments on each word presented in a tachistoscope. The primary independent variable, varied within subjects, was the number of target properties true of each item: $0,1,2$, or 3 (targets). Different property
} 
lists were given to each half of the subjects so that the items with $0,1,2$, or 3 target properties for one half of the subjects had $3,2,1$, or 0 target properties, respectively, for the other subjects. Following an intervening task, there was an unexpected free recall test followed by a recognition memory test with confidence ratings.

Materials. Words were selected from the eight categories defined by the combinations of (consumable or nonconsumable) by (liquid or solid) by (natural or artificial) properties. (Natural was defined for the subjects as items being found in nature in that form.) The eight categories were divided into four groups that had $0,1,2$, or 3 of the target properties. Two opposite sets were chosen as the possible target sets: nonconsumable natural solids (e.g., pebble) and consumable artificial liquids (e.g., bourbon). When one of these categories was used as the target set (3 properties true), the other category items had 0 properties true. The other six categories were combined into two groups of three categories each and had 1 or 2 properties true, depending on which target set was used. Subjects searching for nonconsumable natural solids, then, had items with all 3 properties true (targets), 2 properties true (consumable natural solids, nonconsumable artificial solids, nonconsumable natural liquids), 1 property true (nonconsumable artificial liquids, consumable artificial solids, consumable natural liquids), or 0 properties true (consumable artificial liquids). The other subjects searched for consumable artificial liquids, so these same items had $0,1,2$, and 3 target properties, respectively. Thus, across subjects, the same items appeared in the 0 - and 3-property levels, but these were different from the items in the 1- and 2-property levels. This manipulation was made for ease of item selection. Since two different word sets were used, the levels of memory performance between sets were not interpretable, so each set was analyzed separately.

Thirty words were chosen from each of the four possible groups $(0,1,2$, or 3 target properties). The sets were roughly equated on their frequency of use in text (Kučra \& Francis, 1967). For each subject, 20 words from each set were randomly chosen as the items to be classified. The items were shown in a different random order for each subject. Four primacy and two recency buffers were also included in the classification task.

For the recognition test, half of the items that had been classified in each word set (olds) were presented along with the remaining 10 items from each word set that had not been classified (news). Thus, the distractors were just as likely to have been presented in the classification task as were the items actually presented. The recognition items were printed on three computer sheets, in a random order for each subject, with four possible choices to the right of each word: "Almost sure old," "Maybe old," "Maybe new," and "Almost sure new."

Apparatus. For the classification task, the subjects viewed the words in the bottom field of a modified Siliconix tachistoscope. The words were typed in Gothic capital letters and centered in a $13 \times 7 \mathrm{~cm}$ window at a distance of $51 \mathrm{~cm}$.

Procedure. Subjects were told that there would be several short experiments and that the first one examined the speed with which they could decide whether an item was in a category defined by having three properties. Half of the subjects received one of the target sets, and the other half of the subjects received the other target set. They were told to respond as quickly as possible while maintaining high accuracy. The property meanings were briefly defined on the sheets.

Subjects were seated in front of the tachistoscope. A response box was mounted with three buttons: a black one with a red button to each side. To start a trial, subjects pressed the black button. They then had $1.5 \mathrm{sec}$ before the stimulus was presented to put an index finger on each red button. When the item was shown, subjects pressed one button for a "Yes" response (the item contained all three target properties) and the other button for a "No" response. The assignment of Yes and No buttons was counterbalanced across subjects. After a response, the experimenter wrote down the reaction time and response, changed stimulus cards, and said "Ready." Anytime thereafter, subjects could initiate the next trial. They were allowed to look back at the properties between trials (though after the first few trials, they rarely did). Six practice trials were given, with feedback. After the practice trials, the 86 -item list ( 20 items from each property level and six buffers) was presented without feedback. The classification phase took about $15 \mathrm{~min}$, with a rest halfway through. Short breaks between trials were encouraged, but rarely taken.

Following the classification task, subjects were given six addition and subtraction problems with two three-digit numbers in each. They were asked to solve each problem as quickly as possible, and then to redo each problem more slowly. Including instructions, the task took about $5 \mathrm{~min}$.

Next, there was an unexpected free recall test for items presented in the tachistoscope. Subjects wrote as many list words as they could remember in any order. After $6 \mathrm{~min}$, a line was drawn below the last word recalled. The structure of the list (i.e., the properties and categories used) was then explained, and $3 \mathrm{~min}$ more were given to allow the subjects to use this information to bolster recall. The second recall resulted in only a small number of additional words and could not be analyzed separately. These data were combined with the first recall for all analyses. The final phase was the unexpected test for recognition memory, for which subjects worked at their own pace. They were encouraged to use all the confidence categories.

Subjects. Ten Stanford University students participated for course credit or $\$ 2.50$. The session lasted $1 \mathrm{~h}$ and each subject was tested individually.

\section{Results and Discussion}

Recall. The more target properties a word had, the more likely it was to be recalled. Only words correctly classified (93\%) were included in the analyses, and an arcsin transformation of the proportions recalled was taken. The two word sets $[(0$ and 3$)$ and $(1$ and 2$)$ target properties] were analyzed separately. Only differences within each word set are interpretable in terms of the experimental variable, since recall level differences across word sets may be due to item differences alone. Both sets showed significantly better recall for items with a greater number of target properties. For items with 0 and 3 target properties, the mean proportion recalled was .174 and .385 , respectively $[F(1,9)=17.14, p<.005, \mathrm{MSe}=.067]$. For items with 1 and 2 target properties, the means were .121 and $.247[\mathrm{~F}(1,9)=14.49, \mathrm{p}<.005, \mathrm{MSe}=.038]$.

Recognition. The recognition data presented some difficulties in analysis. The data of interest are the hit rates, since the effect of these hypotheses on false alarms is uncertain [e.g., frequency theory's implicit associative responses (Ekstrand, Wallace, \& Underwood, 1966) might predict that false alarms would increase with hits]. The hope was that the false alarms between property levels would be roughly the same and that $\mathrm{d}^{\prime}$ analyses could be used. Unfortunately, the hit rate was close to perfect $(95 \%)$. Neither $d^{\prime}$ analyses nor converting confidence ratings to numerical values seems appropriate, since these would reflect primarily false alarm differences. ${ }^{1}$ For the subjects whose hit rates were not perfect, the hit rate was higher for the 3 level than for the 0 level ( 5 of $5, p=.03$ ), but the 2 level was 


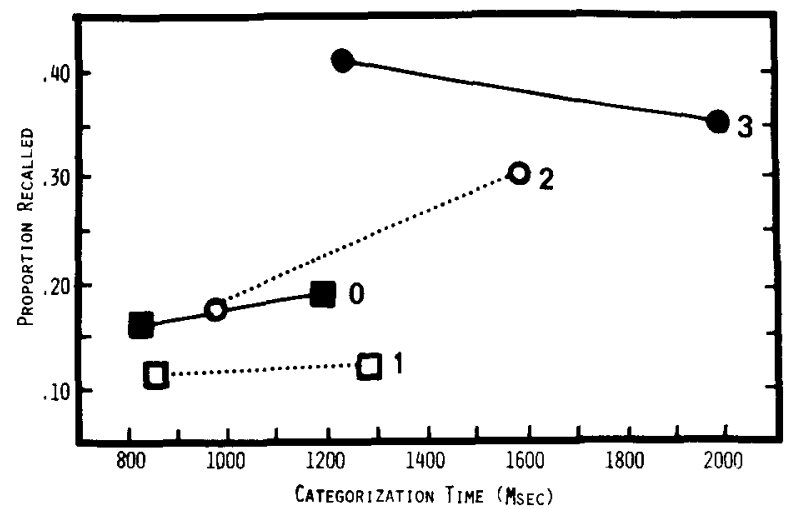

Figure 1. Experiment 1: Proportion recalled for medians of fast and slow processing time decisions within each property level.

not higher than the 1 level ( 2 of 6 ). Though these results are not compelling and this analysis has some problems (e.g., possibly different response bias for the target set), they suggest at least small effects on recognition.

Reaction times. Reaction times were analyzed in a manner similar to that used by Craik and Tulving (1975) in their reanalysis mentioned earlier, although I used recall rather than recognition data. For each subject, using the 20 words from each property level, the 20 reaction times (eliminating errors of classification) were divided into fast and slow halves. For each of these sets, the median time and proportion recalled were calculated. These recall data, with the mean of the medians over subjects, are given in Figure 1, separately for words having $0,1,2$, or 3 target properties ("property level").

As Figure 1 illustrates, within a given property level, longer processing time was not generally associated with greater recall. For the 0,1 , and 3 levels, all $\mathrm{Fs}<1$. For the items with 2 target properties, the trend for increased recall with increased processing time was not significant $[F(1,9)=2.97, \mathrm{p}>.10, \mathrm{MSe}=.150] ;$ only 6 of the 10 subjects showed a difference in this direction. Although processing time within a property level did not affect later recall, the number of target properties substantially affected recall. This effect is most obvious in comparing the slower times for the 0-property level with the faster times for the 3-property level. Although these times were about equal, the proportions recalled were quite different (.190 and .411). (A comparison of 0 and 1 is not meaningful, since they are based on different items.) A similar figure for the recognition data is not included because of the close to perfect performance on the old items (the only items for which reaction times are available).

These results, then, provide evidence that, in this task, while processing time and recall increase with increasing number of decisions, processing time is not a major determinant of recall. ${ }^{2}$ The recall results replicated the findings of Johnson-Laird et al. (1978), while the reaction times within a property level were not predictive of memory performance. The recognition data were difficult to interpret due to ceiling hit rates, but the $0-3$ comparison seems consistent with these findings.

\section{EXPERIMENT 2}

The processing time explanation may be discarded, leaving the retrieval aid and number of positive decisions as alternative hypotheses to the number-of-decisions explanation. The retrieval aid explanation proposes that since subjects have classified items using three properties, at retrieval these properties will be used, either singly or in combination, to aid recall. The recognition memory results in Experiment 1 might have provided some evidence against this notion, since retrieval effects in recognition are generally small, but the results were not compelling. The number-of-positive-decisions hypothesis claims that only properties that match target properties will improve memorability of an item. In Experiment 2, these two possibilities were unconfounded from number of decisions by manipulating the orienting task.

Three groups of subjects, named the ALL, ANY, and EACH groups, were each given different classification instructions. The ALL group, as in Johnson-Laird et al. (1978) and Experiment 1, responded "Yes" if an item had all three target properties and "No" otherwise. The ANY group subjects responded "Yes" if the item contained any of the three properties and "No" if it contained none of them. Subjects in the EACH group recorded three separate responses for each item, a Yes-No decision about each of the three properties. Following this orienting task, all three groups were treated identically, receiving an intervening task, a free recall test, and a recognition memory test. The expected number of decisions in each condition, given a selfterminating search, are given in Table 1. Assuming a random ordering of properties over subjects, the numberof-decisions hypothesis predicts the way the classification instructions will affect recall of words at different property levels. The ANY group should recall in the opposite pattern from the ALL group, since the more target properties an item contains, the sooner one will be found, so fewer decisions will be made. The EACH subjects should show no effect of property level, since all items require the same three decisions in this condition.

This orienting task manipulation unconfounds the remaining hypotheses. If the target properties were used as retrieval cues, one would predict that the more target

Table 1

Expected Number of Decisions for the Three Orienting Tasks of Experiment 2

\begin{tabular}{lcccc} 
& \multicolumn{4}{c}{ Number of Target Properties } \\
\cline { 2 - 5 } Task & 0 & 1 & 2 & 3 \\
\hline ALL & 1.00 & 1.33 & 2.00 & 3.00 \\
ANY & 3.00 & 2.00 & 1.33 & 1.00 \\
EACH & 3.00 & 3.00 & 3.00 & 3.00 \\
\hline
\end{tabular}


Table 2

Expected Number of Positive Decisions for the Three Orienting Tasks of Experiment 2

\begin{tabular}{lcccc}
\hline & \multicolumn{4}{c}{ Number of Target Properties } \\
\cline { 2 - 5 } Task & 0 & 1 & 2 & 3 \\
\hline ALL & .00 & .33 & 1.00 & 3.00 \\
ANY & .00 & 1.00 & 1.00 & 1.00 \\
EACH & .00 & 1.00 & 2.00 & 3.00 \\
\hline
\end{tabular}

properties an item contains, the more likely it is to be remembered. Thus, if the ALL group shows better recall with an increasing number of target properties, then so should the other two groups. Though different retrieval hypotheses might be postulated for each group that would make the same predictions as the number-ofdecisions hypothesis, I could think of no single retrieval hypothesis that could make those predictions.

The expected numbers of positive decisions, assuming a self-terminating search, are given in Table 2 . As Table 2 shows, the predictions based on positive decisions differ greatly from those based on the number of decisions, and differ slightly from the predictions of the retrieval aid hypothesis. The positive-decision hypothesis predicts, generally, increasing memorability with increasing number of target properties, but the predicted relative sizes of the effects across groups should differentiate it from the retrieval aid predictions.

A brief comment may clarify the logic of this study. A serial self-terminating decision rule has been invoked to derive the number-of-decisions and the number-ofpositive-decisions predictions. The self-terminating rule assumption is thus being jointly tested with the numberof-decisions hypothesis; if these predictions are confirmed, the confidence in both should increase. A serial self-terminating search also agrees with the introspections of the author, the subjects of Experiment 1, and the subjects in the study by Johnson-Laird et al. (1978, p. 374). Note, however, that the predicted ordering of conditions in Tables 1 and 2 depend only on the selfterminating aspect, not necessarily on the serial assumption (see Townsend, 1974). The predictions would not change qualitatively if the target properties were checked in a self-terminating parallel model, if the finishing times had some variability.

\section{Method}

The method was similar to that of the first experiment, with some changes, mostly in the classification task.

Design. For the classification task, subjects were given three target properties and a list of items. Three groups of subjects received different instructions. The ALL group received similar instructions to those of Experiment 1 . They were to respond "Yes" if an item had all three target properties and "No" otherwise. The ANY group responded "Yes" if an item had any of the three target properties and "No" if it had none. The EACH group recorded three responses-a "Yes" or "No" concerning whether an item had each of the three target properties. The three groups were treated identically after the classification task. They participated in an intervening task, an unexpected free recall test, and an unexpected recognition memory test.
Materials. The words were the same as in Experiment 1. For the classification phase, items were printed on computer sheets in a random order for each subject. For the ALL and ANY groups, the items were in two columns with a "Yes" and "No" printed to the right of each item. For the EACH group, one item appeared on each line with three sets of "Yes" and "No" printed to its right. At the start of the categorization task, subjects in the EACH group were asked to write one target property at the top of each Yes-No column. For this task, 60 items were presented, 15 from each property level. The recognition test included 10 of the 15 items as "olds" and 10 "news" from the 15 items at each property level not shown in the categorization task.

Procedure. The differences from Experiment 1 were in the classification and intervening tasks. Also, subjects were tested in groups rather than individually. Each subject received a booklet of sheets for the classification and intervening task sheets. Subjects were told that there would be several short experiments, with the first one examining the speed and accuracy of decisions about the properties of words. Accuracy was stressed. The instructions explained the target properties and the task subjects were to perform (ALL, ANY, or EACH). The speed aspect of the classification was made plausible by asking each subject to raise a hand as (s)he finished the classification sheet and to then copy onto the sheet the time that the experimenter wrote on the board. The time ranges for the tasks were 150 $370 \mathrm{sec}$ for ALL, 110-260 sec for ANY, and $390-630 \mathrm{sec}$ for EACH.

Upon completion of the classification task, subjects proceeded to the intervening task, a page of number series problems. Each number series problem consisted of several numbers for which the subjects were to fill in the next number in the series (e.g., $113253749_{\text {_ }}$ ). Subjects worked on the problems until a few minutes after the last subject had completed the classification phase. A second booklet was then distributed which contained the recall and recognition memory test sheets, exactly as in Experiment 1.

Subjects. Forty-eight University of Santa Clara students participated for course credit. Each group was run separately, but within several hours, to minimize the risk of communication about the memory tests. Through variation in sign-up rates for the hours of the three group sessions, there were 15 subjects in the ALL group, 13 in the ANY group, and 20 in the EACH group.

\section{Results and Discussion}

The recall results were very close to those predicted by the number-of-decisions hypothesis, and were inconsistent with the retrieval aid and the number-of-positivedecisions hypotheses. The recognition memory scores showed few differences, due to ceiling effects on the hits.

The classification errors were higher than in the first experiment $(.14, .24$, and .04 for the ALL, ANY, and EACH groups, respectively), but all data were included for recall and recognition analyses. ${ }^{3}$ To the extent that subjects had a truly different classification than the experimenter-intended one, the expected differences in recall would be attenuated. The only data not included in the analyses were those of one subject in the ANY group, because of his unreasonable influence on the results. This subject completed the classification faster than anyone else, recalled more items at Property Level 3 than anyone in all three groups, and tied for most items recalled at Level 2. (These last two results are exactly the opposite of what the combined data of 
the other 12 subjects in the ANY group showed.) Though there are obvious difficulties in excluding a subject on the basis of the results of interest, I believe the extreme influence of his data on the experimental findings warrants such exclusion. The findings within the ANY group including these data are given in a footnote.

Recall. The mean recall for each condition (backtransformed from the arcsin means) is given in Table 3 , separated by word sets. Remember that differences in recall level between word sets are not pertinent to the experimental question. As may be seen, with an increasing number of target properties, recall increased in the ALL group, decreased in the ANY group, and remained relatively constant in the EACH group. The analyses generally confirmed these observations. In examining these results, it should be remembered that the 0 vs. 3 comparison is more diagnostic, that is, the predictions of the proposed explanations differ more for this comparison than for the 1 vs. 2 comparison and the effects are predicted to be larger.

First, the effects within each group will be reported and then the interactions. For the ALL subjects, recall increased significantly from 0 to 3 target properties $[F(1,13)=7.66, p<.02, \mathrm{MSe}=.093]$ and from 1 to 2 target properties $[\mathrm{F}(1,13)=5.45, \mathrm{p}<.05$, MSe $=$ $.041]$. Comparing the pooled low $(0$ and 1$)$ vs. high $(2$ and 3$)$ property levels yields a strong effect $[F(1,13)$ $=17.32, \mathrm{p}<.001, \mathrm{MSe}=.050]$. The ANY results were in the opposite direction, but the effect was not as large. The decrease from 0 to 3 barely missed the .05 significance level $[F(1,10)=4.49, p<.07, \quad \mathrm{MSe}=$ $.146]$, and the decrease from 1 to 2 was not reliable $(\mathrm{F}<1)$. The combined data also showed a trend $[F(1,10)=4.79, p<.06, \mathrm{MSe}=.130] .^{4}$ The $\mathrm{EACH}$ group showed no effect of the number of target properties on recall from 0 to $3(\mathrm{~F}<1)$ but a marginally significant increase from 1 to 2 properties $[F(1,18)=$ $3.25, \mathrm{p}<.10, \mathrm{MSe}=.058]$. Combining the low and high property levels produced no reliable difference $[\mathrm{F}(1,18)=1.84, \mathrm{p}>.15, \mathrm{MSe}=.058]$.

The interactions between property levels and groups were checked to confirm that the effect of the number of target properties was different for each group. Of main interest is the ALL vs. ANY contrast, since opposite results were predicted for these groups. The other two contrasts are included for completeness, but since there are only two degrees of freedom, their interpretation is uncertain. For the 0 vs. 3 target property levels, all interactions are reliable. For the 1 vs. 2 levels, the

Table 3

Proportion Recalled in Experiment 2

\begin{tabular}{lcccc}
\hline & \multicolumn{4}{c}{ Number of Target Properties } \\
\cline { 2 - 5 } Task & 0 & 3 & 1 & 2 \\
\hline ALL & .227 & .377 & .178 & .252 \\
ANY & .367 & .218 & .233 & .182 \\
EACH & .404 & .409 & .209 & .267 \\
\hline
\end{tabular}

differences are in the predicted direction but are often unreliable. The combined results show clear interactions among the groups.

For the ALL-ANY contrast, the 0 vs. 3 interaction was highly reliable $[F(1,23)=11.72, p<.002, \mathrm{MSe}=$ $.125]$. The 1 vs. 2 interaction was smaller $[F(1,23)=$ $2.37, .10<\mathrm{p}<.15, \mathrm{MSe}=.125]$; combining low and high property levels yielded a highly significant interaction $[F(1,23)=17.24, p<.001, \mathrm{MSe}=.085]$.

The ALL-EACH interaction was significant for the 0 vs. 3 levels $[\mathrm{F}(1,31)=4.76, \mathrm{p}<.05, \mathrm{MSe}=.080]$ but not for the 1 vs. 2 test, $(F<1)$. The combined data show the interaction $[F(1,31)=4.35, p<.05$, $\mathrm{MSe}=.055]$.

The ANY-EACH comparison showed a reliable interaction for the 0 vs. 3 contrast $[F(1,28)=4.48$, $\mathrm{p}<.05, \mathrm{MSe}=.097$ ] but not for the 1 vs. 2 test $[F(1,28)=2.13, p>.15, \mathrm{MSe}=.120]$. Combining the low and high property levels yielded a significant interaction $[F(1,28)=8.11, p<.01, \mathrm{MSe}=.084]$.

These recall results, then, are generally consistent with the number-of-decisions predictions. The more diagnostic 0 vs. 3 comparisons showed significantly reliable effects and interactions as predicted, as did the combined high vs. low comparisons. The less diagnostic 1 vs. 2 comparisons were as predicted except for a marginally significant increase in the EACH condition. The relative proportions recalled were as predicted in Table 1, with minor exceptions. The 1 property level in the EACH condition was less than that in the ANY condition (it was predicted to be greater), though the difference was only $2.4 \%$. While no explanation has been offered for this misprediction, it does not seem such a serious violation as to invalidate the other findings.

Recognition memory. The recognition results presented the same difficulties in analysis as those in Experiment 1 . The hit rate was high $(94 \%)$ and many subjects were at the ceiling. ${ }^{5}$ For those subjects whose hit rates were not perfect, a sign test analysis was performed, as in Experiment 1. For the more diagnostic 0 vs. 3 levels, the 3-level hit rate was higher in the ALL condition $(10$ of $13, \mathrm{p}<.05)$, nonsignificantly lower in the ANY condition ( 3 of $10, p<.20$ ), and equal in the EACH condition ( 7 of 13 ). The 1 vs. 2 levels showed no differences.

\section{EXPERIMENT 3}

The recall results of Experiment 2 provide evidence against the number-of-positive-decisions and retrieval explanations. The third experiment replicates the second experiment, with some small modifications. The earlier design with two separate item sets did not allow a direct comparison across all property levels, whereas the design of this experiment does. Two, rather than three, binary properties categorized the items, but all of the four possible property pairs were used as targets. (The item assignment difficulties prohibited the use of three 
properties with this design.) With two target properties, the hypotheses predict the same qualitative pattern as that in Table 1, though the expected differences between conditions generally decrease. Also, different item sets and target properties were used and no recognition test was given.

\section{Method}

Design and Procedure. The classification task design was as that in Experiment 2, except that two target properties were used instead of three. Following the classification task, subjects participated in a number series task for $1 \frac{1 / 2}{2} \mathrm{~min}$ after the last subject finished the classification, and were then given an unexpected free recall test. The time ranges for the classification task were 70-190 sec for ALL, 105-240 sec for ANY, and 120$430 \mathrm{sec}$ for EACH.

Materials. Words were selected from the four categories defined by the combination of (living or dead) and (smaller or larger than a child). (Subjects were told to use a 7-year-old as a child, were shown the approximate height, and told that items would clearly be smaller (larger).] Ten words were selected in each of the four categories, with equal mean frequency (Kucera \& Francis, 1967). The 40 words were randomized and typed, five to a page. The order of the eight pages was randomized for each subject. Primacy and recency pages, with five items each, were also included in the booklet. The four possible target pairs were used equally often.

The 40 critical items were rated on the two properties by a different group of seven subjects to check the classification with no speed pressures. All subjects agreed on 36 of the 40 items, and all but one agreed on the other 4 items.

Subjects. Seventy subjects from Stanford University and the University of Santa Clara participated for course credit in groups of 5 to 16. Through variation in sign-up rates for the sessions, there were 26 subjects in the ALL group, 23 in the ANY group, and 21 in the EACH group. The sessions lasted about $20 \mathrm{~min}$.

\section{Results and Discussion}

The recall results were again very similar to those predicted by the number-of-decisions hypothesis, and were inconsistent with the number-of-positive-decisions and the retrieval aid hypotheses. The classification errors were $.01, .04$, and .03 for the ALL, ANY, and EACH groups, respectively.

The mean recall for each condition (backtransformed from the arcsin means) is given in Table 4 . As can be seen, with an increasing number of target properties, recall increased in the ALL group, decreased in the ANY group, and remained relatively constant in the EACH group. The analyses confirmed these observations. The effects are not as large as the $0-3$ conditions of Experiment 2, but the difference in the expected number of decisions is less with two target properties than with three.

Table 4

Proportion Recalled in Experiment 3

\begin{tabular}{lccc} 
& \multicolumn{3}{c}{ Number of Target Properties } \\
\cline { 2 - 4 } Task & 0 & 1 & 2 \\
\hline ALL & .208 & .266 & .334 \\
ANY & .297 & .280 & .168 \\
EACH & .260 & .277 & .277 \\
\hline
\end{tabular}

The analysis within each group is reported before the interactions. The monotonic effects predicted for the $\operatorname{ALL}(1.0,1.5,2.0)$ and $\operatorname{ANY}(2.0,1.5,1.0)$ groups by the number-of-decisions hypothesis were tested by the 0 vs. 2 comparisons and were reliable for both the ALL group $[\mathrm{F}(1,22)=7.59, \mathrm{p}<.05, \mathrm{MSe}=.107]$ and the ANY group $[F(1,19)=13.14, p<.005, \mathrm{MSe}=.085]$. Since linear trend analyses of three points exclude the middle point, a Newman-Keuls procedure was used to test differences between the three target property levels. In the ALL group (MSe $=.107$ ), the 0 and 2 levels differed, but the 1 level did not significantly differ from either of the others. In the ANY group $(\mathrm{MSe}=.077)$, the 0 and 1 levels differed from the 2 level, but not from each other.

Since the number-of-decisions hypothesis predicts no differences for the EACH group, its effects were tested by three pairwise comparisons to maximize the probability of rejecting the hypothesis. None of the differences was reliable (all Fs $<1$, average $\mathrm{MSe}=.083$ ).

The pairwise interactions were tested as in Experiment 2 to confirm that the effect of the number of target properties is different for each group. The analysis of main interest is the ALL-ANY interaction, which was very reliable $[F(2,82)=10.65, p<.001, \mathrm{MSe}=$ .095]. The ANY-EACH interaction was also significant $[\mathrm{F}(2,72)=4.57, \mathrm{p}<.05, \mathrm{MSe}=.083]$, but the ALLEACH interaction was not $[\mathrm{F}(2,78)=1.34, \mathrm{p}>.25$, $\mathrm{MSe}=.097]$.

These recall results, then, generally favor the numberof-decisions hypothesis. The pattern is very similar to the predicted pattern, though there are slight differences. In the ALL condition, for which the three hypotheses made similar predictions, the 1-level recall did not significantly differ from the other two levels. In the ANY condition, contrary to prediction, the 0 -level recall was not significantly greater than the 1-level recall. Despite these differences in statistical significance from the predicted pattern, the ordering of the levels is exactly as predicted by the number-of-decisions hypothesis in both these conditions, and the EACH condition is very close to the predicted constant recall. Further, while the number-of-decisions hypothesis claims there is a monotonic relation between number of decisions and recall, there is no claim that this relation is linear.

\section{GENERAL DISCUSSION}

The results of these three experiments support the hypothesis that the number of semantic decisions made about a word is a major determinant of its memorability. Experiment 1 showed that, for a fixed number of target properties, longer processing times were not associated with higher recall. In Experiment 2, recall followed predictions from the number-of-decisions hypothesis, except for a marginally significant increase from 1 to 2 properties for the EACH group (where equality was 
predicted). The retrieval aid and number-of-positivedecisions hypotheses were disconfirmed by the results for the ANY and EACH groups. The recognition findings in the first two experiments were generally uninformative, but showed some suggestion of the predicted effects on hits in the 0-3 levels. In Experiment 3, the pattern of recall results was very close to the pattern predicted by the number-of-decisions hypothesis and inconsistent with the other two hypotheses. Additionally, in an earlier pilot study with the materials and design of Experiment 3, similar results were obtained under intentional memory instructions.

The number-of-decisions idea, then, appears to be a useful, noncircular means of studying encoding effects within the semantic domain, and could probably be extended to study effects within other domains as well. Now that the major determinant of an item's memorability in this procedure is more certain, it can be used to investigate issues requiring such a tool. The method does not require complete agreement between the experimenter's and subjects' classifications but disagreements generally would attenuate the expected effect.

\section{Number of Decisions Compared to the Elaboration Hypothesis}

Of the several ideas that have been proposed to augment depth in the levels-of-processing framework, number of decisions corresponds most closely to elaboration. Elaboration refers to the richness of the trace laid down within a single level (breadth rather than depth). Craik (1977) reviewed the evidence and reasoning that had led to the proposal of elaboration, and suggested that the degree of elaboration depends on the compatibility between the context and the item (but only when they are congruous). Johnson-Laird et al. (1978) claimed that the idea of number of decisions (a) accounts for all the results elaboration was proposed to explain, (b) does so while avoiding the problem of assessment, and (c) provides predictions in cases where the elaboration prediction is unclear. For example, Johnson-Laird et al. claimed that deciding about semantically close conjectures (e.g., "A whale is not a fish") would require more processing (number of property decisions) than would deciding about a semantically remote conjecture (e.g., "A heaven is not a fish"), so that subjects would later recall items from close decisions ("whale") more often than those from remote decisions ("heaven"). This same reasoning could be applied to the sentence complexity study by Craik and Tulving (1975, Experiment 7); more complex sentence frames (into which a presented word may or may not fit) require more decisions, perhaps about the increased number of constraints (properties) placed on what the suitable objects might be. Even Schulman's (1974) congruity results could be explained by the number of decisions needed to make a response, rather than invoking his similar interpretation of relational encodings. Schulman's subjects answered queries about attributes or superordinate categories of items (Is a TWINGE sudden?
Is SPINACH ecstatic? Is a CORKSCREW an opener? Is a DUNGEON a scholar?). In an unanticipated test, words in queries producing positive responses were better remembered than ones producing negative responses. Since negative items violated many of the selectional restrictions (Katz \& Fodor, 1963), they might have been answered after very few decisions, while positive items probably required more extensive checking.

Although I agree that the number-of-decisions idea is preferable to the elaboration idea because of its greater specificity, I do not agree with the elaboration derivation of Johnson-Laird et al. (1978). My interpretation of elaboration would predict that, since whale and fish have so many preexisting relations, the trace would be far more elaborated than would the heaven-fish trace. A major difficulty with elaboration is not that it fails to predict the correct results, but that it is sufficiently ambiguous to predict almost any results (as is illustrated by the opposing explanations just derived). In addition, intentional manipulations of elaboration encounter difficulties so that "Pragmatically, elaboration is a slender reed to lean on" (Postman et al., 1978, p. 684).

Perhaps a conceptual difference between (my interpretation of) elaboration and number of decisions is their relative reliance on preexisting relations. ${ }^{6}$ The elaboration of a trace is strongly influenced by the (preexisting) compatibility between the context and the item. The number of decisions will also normally be influenced by the preexisting relations, but need not be. By suitable experimental manipulation (perhaps as in the ANY and EACH conditions of Experiments 2 and 3 ), one may test the influence of congruous preexisting relations and number of decisions on memorability.

\section{A Memory Network Interpretation of Number of Decisions}

This final section contains a spreading activation network interpretation of the number-of-decisions hypothesis. Presentation of the model was motivated by two considerations. Although many models are consistent with these results, presentation of a specific model allows others to assess its plausibility and usefulness for understanding other findings. A network model was chosen rather than a feature-comparison model because Bock (1976) has sketched a model of the latter type for a similar task. Another reason for presenting a specific model is to bridge the gap between levels-of-processing explanations and those offered in memory network theories. Although some network models have attempted to account for the levels-ofprocessing findings (e.g., Anderson, 1976), little interaction has occurred between the two orientations in terms of either shared ideas or data. Hopefully, further demonstrations of their compatibility will promote their rapprochement.

The semantic network model proposed is a blend of the spreading activation, intersecting search ideas of ACT (Anderson, 1976) and the list context and recall 


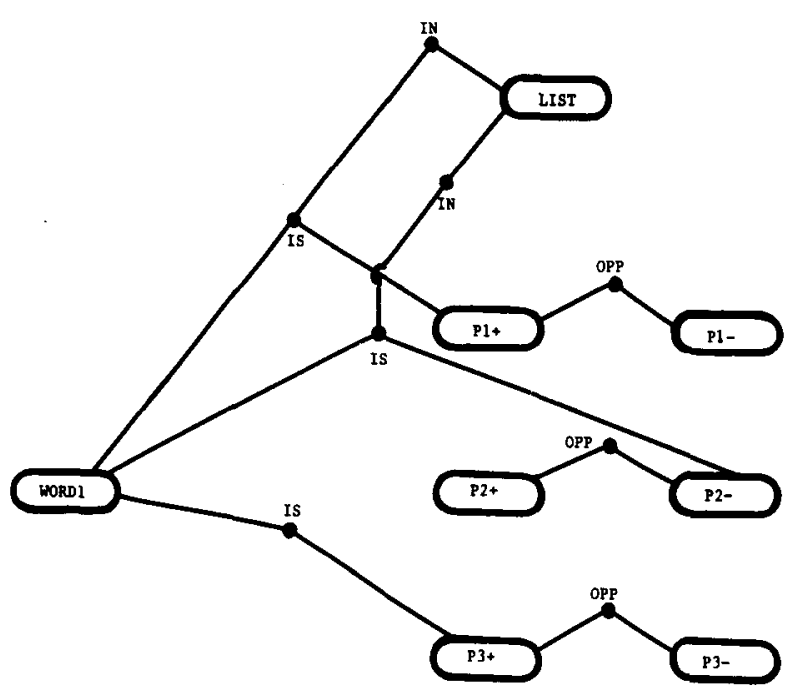

Figure 2. A schematic network representation of a concept (WORD1), the target properties (P1+, P2+, P3+), their contrasting properties (P1-, P2-, P3-), the preexperimental links between the concept and the properties, and the context (LIST) tagging of the two propositions which were checked.

ideas of HAM (Anderson \& Bower, 1973, 1974). Memory is represented as an associative network of propositions interconnecting concepts. The relations between concepts are encoded in propositional structures connecting the concept nodes. (Let us ignore for this discussion the difference between a concept and a word that symbolizes it.)

Assume that each word (concept) node is linked preexperimentally to a target property (e.g., bourbon is liquid) or to a contrary property from which a "No" decision may be inferred (e.g., bourbon is liquid implies a "No" to a query about whether bourbon is solid). Experimental presentation of an item is assumed to have the following effect. For each target property checked for that word, the relevant association is strengthened, and this information is tagged (with a list-context proposition) as having occurred in the experiment (Anderson \& Bower, 1974). If a preexperimental property connection is used to infer that the item does not have the target property being checked, the preexperimental association is strengthened and tagged. Figure 2 contains a schematic network for a word having two target properties. If subjects in the ALL group categorized the word by checking the properties in the order $\mathrm{P} 1, \mathrm{P} 2$, and $\mathrm{P} 3$, the preexperimental connections to the first target property and the second contrasting property would be strengthened and tagged. (Assuming a selfterminating model, the third property would not be checked.)

At the time of free recall, the subject may follow one of several possible retrieval "strategies." The simplest strategy, and one generally consistent with the results, is that the experimental context node is activated and the activation spreads through all the associative links attached to the node. The more properties of an item that are checked, the more links by which it is connected to this context node and, hence, the more likely it is to be recalled. Referring to Figure 2, activating the context node would result in the two tagged propositions being activated. Thus, the number of decisions made about an item affects later recall by increasing the number of strengthened connections from the context node to the item node. It is the number of decisions that determines later recall, not the number of positive decisions or target properties.

Certainly, other retrieval strategies could produce similar results, but many others would lead to predictions inconsistent with the data. I have tried to present just one means in which the effect of number of decisions could be due to differential tagging of concepts at encoding. This general tagging notion also implies (with some auxillary assumptions) a possible explanation for why an additional decision on an item improves memorability less if it is similar to an earlier decision on that item (e.g., Klein \& Saltz, 1976). A highly similar decision is likely to use (and strengthen) the same propositional information, which may not be as effective for later recall of that item as a decision using different propositional information.

In conclusion, these experiments have unconfounded the number-of-decisions idea from several possible hypotheses and found that the number of semantic decisions made about a word is the major determinant of its memorability. This idea seems to correspond closely to the principle of elaboration within the levels. of-processing framework, but it is not vulnerable to the criticisms directed at elaboration. Hopefully, these results will encourage others to use number of decisions as a guide in investigating encoding effects within levels of orienting tasks.

\section{REFERENCES}

Anderson, J. R. Language, memory, and thought. Hillsdale, N.J: Erlbaum, 1976.

Anderson, J. R., \& Bower, G. H. Human associative memory. Washington, D.C: Winston, 1973.

Anderson, J. R., \& Bower, G. H. A propositional theory of recognition memory. Memory \& Cognition, 1974, 2, 406-412.

BADDELEY, A. The trouble with levels: A reexamination of Craik and Lockhart's framework for memory research. Psychological Review, 1978, 85, 139-152.

Bock, M. The influence of instructions on feature selection in semantic memory. Journal of Verbal Learning and Verbal Behavior, 1976, 15, 183-191.

CraIK, F. I. M. Depth of processing in recall and recognition. In S. Dornič (Ed.), Attention and performance VI. Hillsdale, N.J: Erlbaum, 1977.

Craik, F. I. M., \& Lockilart, R. S. Levels of processing: A framework for memory research. Journal of Verbal Learning and Verbal Behavior, 1972, 11, 671-684.

Craik, F. I. M., \& Tulving, E. Depth of processing and the retention of words in episodic memory. Journal of Experimental Psychology: General, 1975, 104, 268-294.

Ekstrand, B. R., Wallace, W. P., \& Underwood, B. J. A frequency theory of verbal-discrimination learning. Psychological Review, 1966, 73, 566-578.

Fisher, R. P., \& Craik, F. I. M. The interaction between encod- 
ing and retrieval operations in cued recall. Journal of Experimental Psychology: Human Learning and Memory, 1977, 3, 701-711.

Frase, L. T., \& Kammann, R. Effects of search criterion upon unanticipated free recall of categorically related words. Memory \& Cognition, 1974, 2, 181-184

HYDF, T. S. Differential effect of effort and type of orienting task on recall and organization of highly associated words. Journal of Experimental Psychology, 1973, 97, 111-113.

Johnson-Laind, P. N., \& Bethrll-Fox, C. E. Memory for questions and amount of processing. Memory \& Cognition, $1978,6,496-501$.

Johnson-Laird, P. N., Gibis, G., \& df:Mowbray, J. Meaning, amount of processing, and memory for words. Memort \& Cognition, 1978, 6, 372-375.

Katz, J., \& Fonon, J. The structure of a semantic theory Language, 1963, 39, 170-210.

KL.\&in, K., \& Sal,Tz, E. Specifying the mechanisms in a levelsof-processing approach to memory. Journal of Experimental Psychology: Human Learning and Memory, 1976, 2, 671-679.

Kućera, H., \& Francis, W. N. Computational analvsis of present-dav English. Providence, R.I: Brown University Press, 1967.

Mandefk, G. Organization and memory. In K. W. Spence \& J. T. Spence (Eds.), The psychology of learning and motivation. New York: Academic Press, 1967.

Moscovitch, M., \& Cralk, F. I. M. Depth of processing, retrieval cues, and uniqueness of encoding as factors in recall. Journal of Verbal Learning and Verbal Behavior, 1976, 15, 447-458.

Nelson, T. O. Repetition and the depth of processing. Journal of Verbal Learning and Verbal Behavior, 1977, 16, 151-171.

Postman, L., \& Kruesi, E. The influence of orienting tasks on the encoding and recall of words. Journal of Verbal Learning and Verbal Behavior, 1977, 16, 353-369.

Postman, L., Thomprins, B. A., \& Gray, W. D. The interpretation of encoding effects in retention. Journal of Verbal Learning and Verbal Behavior, 1978, 17, 681-706.

Rips, L. J., Shoben, E. J., \& Smith, E. E. Semantic distance and the verification of semantic relations. Journal of Verbal Learning and Verbal Behavior, 1973, 12, 1-20.
Schulman, A. I. Memory for words recently classified. Memory \& Cognition, 1974, 2, 47-52.

TownSEND, J. T. Issues and models concerning the processing of a finite number of inputs. In B. H. Kantowitz (Ed.), Human information processing: Tutorials in performance and cognition. Hillsdale, N.J: Erlbaum, 1974.

\section{NOTES}

1. In preliminary data work, these analyses were conducted, and both showed increases from the 0 level to the 3 level but no differences between the 1 and 2 levels. Due to the ceiling hit rate, the $\mathrm{d}^{\prime}$ analyses were modified by setting perfect scores equal to $1-1 /(2 N)$, where $N$ is the number of items. The confidence ratings were assigned values of $1,2,3$, or 4 for increasingly accurate ratings for both old and new items (e.g., a 3 for "Maybe old" rating of an old item).

2. The median split procedure might lead to item selection problems, with the difficult items being in the slow half and masking the effect of processing time. This possibility cannot be refuted, because it could rely on subject-item interactions for which no measure independent of reaction time is available.

3. It seemed preferable to include all the data to avoid item selection problems. In the first experiment, errors were excluded because, with a speeded response, subjects would often comment on their fast errors and, thus, were likely to remember those items.

4. With the outlier subject, the ANY group showed insignificant effects, though the means were in the predicted direction. For the $0-3$ contrast and the combined contrast, there were major changes $[\mathrm{F}(1,11)=1.49, \mathrm{p}>.20, \mathrm{MSe}=.203$ and $\mathrm{F}(1,11)=1.74, \mathrm{p}>.20, \mathrm{MSe}=.204$, respectively $]$. The 1 vs. 2 contrast still had $\mathrm{F}<1$.

5 . By both the modified $d^{\prime}$ and rating analyses, the only significant effect was in the ALL condition, with the 3-level recall greater than the 0 -level recall.

6. I thank P. Johnson-Laird for discussion of this point.

(Received for publication May 5, 1980; revision accepted July 30,1980 .) 